\title{
Site of Care for COVID-19-Like Respiratory Illnesses
}

\author{
John M. Westfall, MD, MPH, Anuradha Jetty, MPH, Stephen Petterson, PhD, and \\ Yalda Jabbarpour, $M D$
}

COVID-19 is primarily a respiratory illness. Historically, upper and lower respiratory illness has been cared for at home or in the ambulatory primary care setting. It is likely that patients experiencing CoVID19-like symptoms may first contact their primary care provider. The Medical Expenditure Panel Survey (MEPS) is a representative sample of patients from the United States that regularly assesses their use of medical care services. We analyzed 2017 MEPS data to determine the number and proportion of patients who were seen in primary care or family medicine ambulatory settings or hospitalized for upper or lower respiratory illness or pneumonia. In a given year, 19.5 million patients are seen by primary care for an upper respiratory illness, 10.7 million patients for bronchitis, and 9 million for pneumonia. In contrast, 890,000 patients are hospitalized with pneumonia. Given that a primary etiology for respiratory illness in early 2020 was SARS CoV-2 (COVID-19), primary care practices likely were the site of first contact for most patients with COVID-19 illness. Unfortunately, there has been inadequate support for in-person and telehealth visits. Primary care clinicians reported serious shortages of personal protective equipment (PPE) and testing capacity. Inadequate reimbursement for telehealth visits coupled with decreased in-person visits put primary care practices at risk of layoffs and closure. Policies related to primary care payment, federal relief efforts, PPE access, testing and follow-up capacity, and telehealth technical support are essential so primary care can provide first contact and continuity for their patients and communities throughout the COVID-19 pandemic response and recovery. (J Am Board Fam Med 2021;34:S26-S28.)

Keywords: Ambulatory Care, Bronchitis, Family Medicine, Pandemics, Personal Protective Equipment, Pneumonia, Primary Health Care, Surveys and Questionnaires, Telemedicine

Is COVID-19 Like Other Respiratory Illness? COVID-19 is primarily a respiratory illness. ${ }^{1,2}$ Although there are additional symptoms related to gastrointestinal disturbance, coagulopathy, dermatologic manifestations, and occasional neurologic components, most cases of COVID-19 are upper and lower respiratory conditions. Historically, upper and lower respiratory (LRI) illness has been cared for at home or in the ambulatory primary care setting. ${ }^{3}$ At the height of the COVID-19 pandemic in the United States, fewer than 5\% of emergency room visits were for COVID-19-like illness (CLI). ${ }^{4}$ The cumulative hospitalization rate for CLI was 29.2 per

This article was externally peer reviewed.

Submitted 8 May 2020; revised 11 June 2020; accepted 25 June 2020.

From the Robert Graham Center, American Academy of Family Physicians, Washington, DC.

Funding: None.

Conflict of interest: All authors declare no conflicts of interest.

Corresponding author: John M. Westfall, MD, MPH, The Robert Graham Center, 1133 Connecticut Ave NW, Suite 1100, Washington, DC 20026 (E-mail: jwestfall@aafp.org).
100,000 , accounting for approximately 100,000 hospitalizations. Given the wide variation in clinical severity, from mild upper respiratory (URI) symptoms with fever and cough, to moderate LRI symptoms of cough and shortness of breath, to severe respiratory distress, it is reasonable to predict that even in the midst of a COVID-19 surge, a vast majority of cases will be identified by primary care clinicians in ambulatory settings through virtual video or telephone encounters or in-person visits.

\section{Where Do Patients with Respiratory Illness} Receive Care?

The Medical Expenditure Panel Survey (MEPS) is a representative sample of patients from the United States that regularly assesses their use of medical care services. ${ }^{5}$ We analyzed 2017 MEPS data to determine the number and proportion of patients who were seen in primary care ambulatory settings or hospitalized for upper or lower respiratory illness or pneumonia. For this analysis, we used a standard 
Figure 1. Respiratory illness and site of care—patients—Medical Expenditure Panel Survey (MEPS) 2017. Abbreviations: LRI, lower respiratory illness; URI, upper respiratory illness.

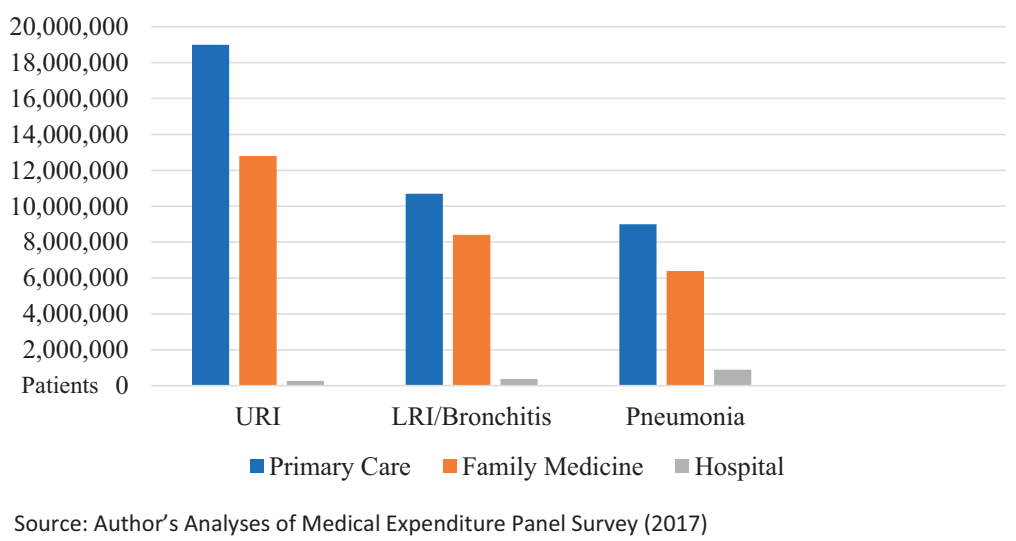

definition of primary care that includes family medicine, general internal medicine, pediatrics, and geriatrics. Because family medicine is often the major or only primary care physician in a community, we analyzed the subset of cases seen in family medicine ambulatory clinics. We also calculated the number of visits to primary care and family medicine physicians.

\section{Primary Care}

In a given year, 19.5 million patients are seen by primary care for a URI and 64 million primary care visits include a diagnosis of URI, 10.7 million patients for LRI/bronchitis and 82.5 million primary care visits include a diagnosis of LRI/bronchitis, and 9 million patients for pneumonia and 38 million primary care visits include a diagnosis of pneumonia. See Figure 1 and Table 1.

\section{Family Medicine}

In a year, 12.8 million patients see a family medicine physician for URI (37.8 million family medicine visits), 8.4 million patients for LRI/bronchitis (62.4 million family medicine visits), and 6.4 million patients for pneumonia (26.2 million family medicine visits).

\section{Hospital}

Each year, 263,000 patients are hospitalized for URI, 372,000 are hospitalized for LRI, and 890,000 are hospitalized for pneumonia. Approximately 416,000 hospitalizations include the diagnosis of URI, 888,000 include the diagnosis of LRI, and 1.5 million include the diagnosis of pneumonia. See Table 2.

\section{What Are the Primary Care Implications for COVID-19?}

The vast majority of patients with respiratory illness who seek care do so in the primary care setting. Even patients with pneumonia are most frequently seen in primary care clinics. These data do not include patients who experience symptoms of a URI but do not seek medical care. It is uncommon for patients with severe respiratory illness to not seek any medical care, so these data likely represent most of the moderate and severe respiratory illness. Given that a primary etiology for respiratory illness in early 2020 was SARS CoV-2 (COVID-19), primary care practices likely were the site of first contact for many patients with COVID-19 illness.

Table 1. Ambulatory Primary Care and Family Medicine Visits for Respiratory Illness

\begin{tabular}{|c|c|c|c|c|c|c|c|}
\hline \multicolumn{6}{|c|}{ Patient Level-Number of Nonduplicated Patients Reporting This Diagnosis } & \multicolumn{2}{|c|}{$\begin{array}{l}\text { Visit Level - Number of Visits } \\
\text { That Included This Diagnosis }\end{array}$} \\
\hline Diagnosis & Primary Care & & Family Medicine & & US Population & Primary Care & Family Medicine \\
\hline & \# Patients & $\%$ & \# Patients & $\%$ & & \# Visits & \# Visits \\
\hline URI & $19,539,206$ & 6 & $12,837,089$ & 4 & $323,141,733$ & $64,101,442$ & $37,873,394$ \\
\hline LRI & $10,728,045$ & 3 & $8,437,528$ & 3 & $323,141,733$ & $82,592,703$ & $62,423,705$ \\
\hline Pneumonia & $9,060,753$ & 3 & $6,479,655$ & 2 & $323,141,733$ & $38,603,646$ & $26,294,553$ \\
\hline
\end{tabular}

Source: Author's analyses of Medical Expenditure Panel Survey (2017).

Abbreviations: LRI, lower respiratory illness; URI, upper respiratory illness. 
Table 2. Hospitalizations for URI/LRI/Pneumonia

\begin{tabular}{|c|c|c|c|c|}
\hline \multicolumn{4}{|c|}{$\begin{array}{l}\text { Patient Level-Number of Nonduplicated } \\
\text { Patients Reporting This Diagnosis } \\
\end{array}$} & \multirow{2}{*}{$\begin{array}{c}\text { Visit Level- } \\
\text { Number of } \\
\text { Visits That } \\
\text { Included This } \\
\text { Diagnosis } \\
\# \\
\text { Hospitalizations }\end{array}$} \\
\hline Diagnosis & $\begin{array}{c}\# \\
\text { Hospitalized }\end{array}$ & $\%$ & $\begin{array}{c}\text { US } \\
\text { Population }\end{array}$ & \\
\hline URI & 263,533 & 0.08 & $323,141,733$ & 416,724 \\
\hline LRI & 372,694 & 0.12 & $323,141,733$ & 888,224 \\
\hline Pneumonia & 891,718 & 0.28 & $323,141,733$ & $1,562,488$ \\
\hline
\end{tabular}

Source: Author's analyses of Medical Expenditure Panel Survey (2017).

Abbreviations: LRI, lower respiratory illness; URI, upper respiratory illness.

Physical distancing coupled with patient fear and uncertainty may have changed the way patients contact their primary care provider. While facing an increase in patient need, the way care is delivered has shifted, with a significant amount provided by telehealth and a steady need for in-person care as well. Etz and colleagues found that primary care practices used a mixture of video, telephone, and in-person visits, but $84 \%$ of clinicians reported they have patients who cannot use telehealth options for care. ${ }^{6}$ Unfortunately, there has been inadequate support for both in-person visits and telehealth visits. Primary care clinicians reported serious shortages of personal protective equipment (PPE) and testing capacity. Even after the Centers for Medicare and Medicaid Services relaxed the rules for telehealth reimbursement, inadequate reimbursement for telehealth visits coupled with decreased in-person visits put primary care practices at risk of layoffs and closure. ${ }^{8,9}$

Primary care plays a prominent role in care for patients suffering respiratory illness. In the COVID19 pandemic, primary care may continue to play an important frontline role in diagnosing COVID-19 infection, providing care and treatment, and offering crucial triage for patients with moderate to severe disease. Preliminary reports from the Centers for Medicare and Medicaid Services indicate that, even among Medicare beneficiaries, two thirds of confirmed COVID-19 cases did not require hospitalization and received their care in ambulatory settings. ${ }^{10}$ Primary care providers have offered a mix of in-person and virtual care to patients with all types of presenting complaints, including URI, a portion of which is likely to be CLI. Primary care will play an important role in the recovery process by identifying patients at risk for COVID-19 and those who may have COVID-19 immunity and by continuing to provide care to patients with the usual acute, chronic, and preventive care needs. Policies related to primary care payment, federal relief efforts, PPE access, testing and follow-up capacity, and telehealth technical support are essential so primary care can provide first contact and continuity for their patients and communities throughout the COVID-19 pandemic response and recovery.

To see this article online, please go to: http://jabfm.org/content/ 34/Supplement/S26.full.

\section{References}

1. World Health Organization. Coronavirus. 2020. Available from: https://www.who.int/health-topics/ coronavirus\#tab=tab_1. Accessed April 29, 2020.

2. Centers for Disease Control. Coronavirus disease 2019 (COVID-19): symptoms. Available from: https:// www.cdc.gov/coronavirus/2019-ncov/symptomstesting/symptoms.html. Accessed April 29, 2020.

3. Renati S, Linder JA. Necessity of office visits for acute respiratory infections in primary care. Fam Pract 2016;33:312-7.

4. CDC National Surveillance Program. Emergency department utilization for COVID-19-like illness. Available from: https://www.cdc.gov/coronavirus/2019ncov/covid-data/covidview/index.html. Accessed April 27, 2020.

5. Medical Expenditure Panel Survey. Available from: https://www.meps.ahrq.gov/mepsweb/. Accessed April 20, 2020.

6. Etz R. Quick COVID-19 primary care surveyweek 8 results. Available from: https://www.greencenter.org/. Accessed June 8, 2020.

7. Etz R. Quick COVID-19 primary care surveyweek 6 results. Available from: https://www.greencenter.org/. Accessed April 29, 2020.

8. Nease D. COVID-19 outreach and resources-working side-by-side with the community. SNOCAP PBRN COVID-19 Survey-Report \#4. Available from: https://medschool.cuanschutz.edu/family-medicine/ community/practice-based-research-networks/covidwork-and-resources. Accessed June 8, 2020.

9. Carrozza M. Coronavirus pandemic-related potential family medicine office closures and economic impact, April through June 2020. 2020. Available from: https://healthlandscape.org/covid19/. Accessed April 29, 2020.

10. Centers for Medicare and Medicaid Services. Preliminary Medicare COVID-19 data snapshot: services January 1 to May 16. Available from: 2020. https:/www.cms.gov/files/document/medicare-covid19-data-snapshot-fact-sheet.pdf. Accessed June 23, 2020. 